\title{
ÍNDICE DE PEGAMENTO DE FRUTOS EM GOIABEIRAS ${ }^{1}$
}

\author{
MÁRCIO CLEBER DE MEDEIROS CORRÊA², RENATO DE MELLO PRADO², WILLIAM NATALE², \\ MARCOS ANTONIO CAMACHO DA SILVA 4 , LUCIANO PEREIRA ${ }^{5}$
}

\begin{abstract}
RESUMO - A produção das plantas está relacionada com o florescimento e frutificação, podendo variar em função de fatores genéticos, ambientais e de manejo dos pomares. Informações dessa natureza, tal como índice de pegamento de frutos, são importantes na caracterização biológica, estudos comparativos de cultivares e predição de safra. O objetivo deste estudo foi determinar o índice de pegamento de frutos em goiabeiras adultas das cultivares Pedro Sato, Paluma e Rica, em pomares comerciais de elevada produtividade, localizados no município de Taquaritinga-SP. No auge do florescimento de cada cultivar ('Pedro Sato': final de fevereiro; 'Paluma' e 'Rica': final de outubro), foram marcados ramos em volta das plantas, à altura do terço médio da copa e contados o número de botões, flores e frutinhos presentes. A operação repetiu-se cerca de 30 dias depois. A contagem final de frutos foi feita quando estes apresentavam diâmetro $\geq 3 \mathrm{~cm}$ nas plantas de 'Pedro Sato' e uma semana antes da colheita nas plantas de 'Paluma' e 'Rica'. A partir do número total de botões florais emitidos (NB) e do número de frutos fixados (NF), calculou-se o índice de pegamento de frutos $[\mathrm{IP}=(\mathrm{NF} / \mathrm{NB}) 100]$. A cultivar Pedro Sato apresentou IP=32,3\%. As cultivares Paluma e Rica apresentaram IP de $18,7 \%$ e $12,2 \%$, respectivamente.

Termos de Indexação: frutificação, retenção, fixação, Psidium guajava, frutífera, fruta.
\end{abstract}

\section{INDEX OF FIXATION OF FRUITS IN GUAVA TREES}

ABSTRACT - Plant production is related with flowering and fruit set and it varies with genetic and environmental factors and handling of the orchards. Information about fruit set are important in the biological characterization, cultivars comparative studies and crop prediction. It was studied the index of fixation of fruits in adult guava trees of Pedro Sato, Paluma and Rica cultivars grown in orchards with commercial high productivity (70$100 \mathrm{tha}^{-1}$ ), located in Taquaritinga, SP, Brazil (21⒉' S; 48 $29^{\prime}$ 'W; 521m). In the peak of flowering of each cultivar ('Pedro Sato': end of February; 'Paluma' and 'Rica': end of October) branches were marked around the plants, in the medium third of the canopy, and the number of present buttons, flowers and little fruits was counted. The operation was repeated about 30 days later. The final counting of fruits was made when they presented $\geq 3 \mathrm{~cm}$ diameter in plants of 'Pedro Sato', and one week before the harvest in plants of 'Paluma' and 'Rica'. The index of fixation of fruits was calculated with the total number of emitted floral buttons (NB) and fixed fruits (NF), [IP=(NF/NB) 100]. The cv. Pedro Sato presented IP=32.3\%. The cv. Paluma and Rica presented IP of $18.7 \%$ and $12.2 \%$ respectively.

Index terms: fruit set, retention, Psidium guajava, fruit tree, fruit.

A goiabeira (Psidium guajava L.) é uma planta cujo cultivo apresenta grande importância econômica e social para o Brasil, especialmente nos estados de São Paulo (68,3 \%) e Pernambuco (10,3 \%), responsáveis por 78,6\% das 260 mil t da fruta produzidas no País em 1998 (AgRiAnUAL, 2001). Em pomares antigos, predominam plantas propagadas via semente; entretanto, nas últimas décadas, tem-se utilizado a propagação vegetativa de cultivares mais adequadas à produção comercial, obtidas em programas de melhoramento genético desenvolvidos no Brasil. Dentre estas, pode-se citar as cultivares Pedro Sato, Rica e Paluma, de aptidão mista, muito utilizadas para renovação ou implantação de novos pomares, com destino prioritário à indústria, especialmente as duas últimas, sendo a 'Paluma' a mais difundida no País (Kavati, 1996).

O comportamento das plantas quanto ao florescimento e frutificação pode variar em função de uma série de fatores (genéticos, ambientais e de manejo dos pomares), tais como tipo e posição das flores nos ramos, deiscência da antera, clima, solo, espaçamento entre plantas, estado nutricional, poda, dentre outros (Erickson, 1968; Rocha et al., 1990; Pereira, 1995; Davies \& Albrigo, 1994; Agustí, 1999; Araújo et al. 1999; Jutamanee et al., 2000). Dada essa variação, torna-se importante o estudo do tema, a fim de definir padrões para conjuntos de combinações dos fatores citados. Cameron \& Frost (1968) chamam a atenção para a importância da observação do comportamento de uma mesma variedade em diferentes ambientes, com o objetivo de avaliar adequadamente suas características genéticas.

A produção das plantas está intimamente relacionada ao florescimento e frutificação. Conforme Forshey \& Elfving (1977) e Dennis Jr. (1981), a produção é função da densidade de plantio, densidade de flores, índice de pegamento de frutos e tamanho dos frutos. De modo geral, para todas as espécies, variedades e condições climáticas, a porcentagem de flores que vingam numa planta, diminui com o aumento da densidade de flores; o tamanho do fruto está inversamente relacionado à densidade de frutos (Forshey \& Elfving, 1977; Dennis Jr., 1981; Agustí et al, 1982; Natale, 1993; Gravina et al., 1996).

$\mathrm{Na}$ literatura, tem-se registrado sensíveis variações no índice de pegamento de frutos para espécies e cultivares distintas. Em macieiras, Dennis Jr. (1986) relata variação de 5 a $15 \%$ no pegamento de frutos; nos cítrus observa-se de $10^{4}$ a $2,5 \times 10^{5}$ flores lançadas por planta em uma safra, conforme a cultivar, localização e estação, e um índice de pegamento oscilando entre 0,1 e $6 \%$ do total de flores emitidas (Goldschmidt \& Monselise, 1977; Agustí et al., 1982; Gravina et al., 1996). Em mangueira, esse índice não chega a 0,01 \% (Simão, 1980). Para goiabeiras, há poucos relatos quanto à retenção final de frutos, com registros de valores de $6 \mathrm{a}$ $20 \%$ (Singh \& Sehgal, 1968; Feldberg et al., 1997).

Informações acerca do florescimento e frutificação das plantas têm importância na caracterização biológica e em estudos comparativos de variedades, nos quais são considerados o potencial de produção e a qualidade do fruto produzido (Doni, 1974; Simão, 1980; Araujo, 2000), características essas relacionadas à porcentagem de flores que são convertidas em frutos maduros (índice de pegamento de frutos).

Segundo Alcázar (2000), o desenvolvimento de programas de melhoramento genético em frutíferas perenes depende da adequada avaliação de variedades disponíveis e, dentre as características consideradas mais importantes a serem avaliadas, está a frutificação efetiva nas plantas. Lopes (1997) evidencia a necessidade de conhecer-se e preservar-se os recursos biológicos, o que mantém a diversidade e permite seu aproveitamento futuro.

Além disso, o índice de pegamento de frutos pode ser usado como um indicativo precoce da produção e tem sido incorporado como componente de modelos matemáticos para predição de produtividade e qualidade (tamanho) de frutos, com objetivo de gerar, com antecedência, informações úteis para cotação de preços, preparativos logísticos e

1 (Trabalho 010/2002). Recebido: 31/01/2002. Aceito para publicação: 25/06/2002.

2 Doutorando, Departamento de Solos e Adubos, FCAV/Unesp, campus Jaboticabal. E-mail: mcleber@fcav.unesp.br. Bolsista da FAPESP.

3 Professor Adjunto, Departamento de Solos e Adubos, FCAV/Unesp, campus Jaboticabal. Via de Acesso Prof. Paulo Donato Castellane, s/n., 14870-000, Jaboticabal SP, Brasil. E-mail: natale@ fcav.unesp.br. Bolsista do CNPq.

4 Mestrando, Departamento de Solos e Adubos, FCAV/Unesp, campus Jaboticabal. Bolsista da FAPESP.

5 Acadêmico do curso de Agronomia, FCAV/Unesp, campus Jaboticabal. 
comercialização da safra (Bustan et al., 1999; Marcelis e Heuvelink, 1999).

Em pomares comerciais de goiabeiras, a queda de frutos pode significar redução da receita ou mesmo prejuízo econômico para o produtor. Diante da perspectiva ou constatação de queda acentuada de flores e frutinhos, em função da ocorrência de pragas, moléstias ou temperaturas extremas, há a possibilidade de adequação do manejo do pomar. Assim, pode-se racionalizar o uso de insumos agrícolas, especialmente fertilizantes, cuja aplicação é feita total ou parcialmente depois do florescimento das plantas, sendo possível redução nas doses, compatibilizando-as com a menor produção esperada.

Diante do exposto, torna-se oportuno o conhecimento do comportamento de goiabeiras cultivadas no Brasil, com vistas ao manejo dos pomares da forma mais econômica possível. Assim, com este estudo, objetivou-se avaliar as cultivares Pedro Sato, Paluma e Rica quanto ao índice de pegamento de frutos, em pomares comerciais adultos, instalados no município de Taquaritinga, São Paulo.

O estudo foi desenvolvido em pomares de goiabeiras adultas, já em plena capacidade produtiva, explorados comercialmente e apresentando elevada produtividade anual $\left(70\right.$ - $\left.100 \mathrm{t} \mathrm{ha}^{-1}\right)$. O solo da área é um Argissolo Vermelho-Amarelo, textura média, localizado no município de Taquaritinga - SP $\left(21^{\circ} 24^{\prime}\right.$ de latitude Sul, $48^{\circ} 29^{\prime}$ de longitude Oeste e altitude de $521 \mathrm{~m}$ ). O clima da região é do tipo Cwa, de acordo com a classificação de Köppen, com precipitação pluviométrica em torno de $1350 \mathrm{~mm}$ por ano, distribuídos em média da seguinte forma: 229; 262; $158 ; 60 ; 51 ; 17 ; 22 ; 33 ; 73 ; 98 ; 111 ; 236 \mathrm{~mm}$ de chuva, respectivamente, de janeiro a dezembro dos anos de estudo.

O espaçamento das plantas nos pomares era de 7 metros entre linhas e 5 metros entre árvores. O manejo fitossanitário foi realizado periodicamente, de acordo com as recomendações técnicas da região, e o manejo da adubação conforme Natale et al. (1996).

As avaliações foram realizadas entre os anos de 1999 e 2001. Para as cultivares Paluma e Rica, foram feitas duas avaliações, uma em 1999-2000 e outra em 2000-2001. Na primeira avaliação, tomaram-se 25 plantas da cv. Paluma e 8 da cv. Rica e, na segunda avaliação, 8 plantas de cada cultivar. Para a cv. Pedro Sato, foi feita uma avaliação, no ano de 2000, tomando-se 29 plantas.

Nos pomares de 'Paluma' e 'Rica' (ambas com cerca de 9 anos de idade no início do estudo), as plantas foram submetidas à poda de frutificação em julho/agosto e alcançaram o ápice do florescimento em outubro/novembro, nos dois anos agrícolas considerados (1999-2000 e 2000-2001). No pomar de 'Pedro Sato' (aproximadamente 7 anos de idade no início do estudo), a poda ocorreu em janeiro e o ápice do florescimento no final de fevereiro/2000

No auge do florescimento, foram marcados ramos à altura do terço médio da copa das plantas. Na primeira avaliação das cvs. Paluma e Rica e na avaliação da cv. Pedro Sato, foram marcados dois "ramos podados" por planta, um de cada lado, voltados para a entrelinha. Esses ramos apresentavam comprimento entre 50 e $80 \mathrm{~cm}$ e número variado de brotações novas (ramos novos, produtivos). Já na segunda avaliação das cvs. Paluma e Rica, foram marcados dez "ramos novos" por planta, sendo que em quatro plantas marcou-se o ramo brotado na primeira gema e, nas outras quatro, o ramo brotado na segunda gema vegetativa, a partir da extremidade do ramo podado, em ambas as cultivares.

Durante as marcações, contaram-se o número de botões, flores e frutinhos por ramo, bem como eventuais cicatrizes ou resquícios de tais estruturas já perdidas. Cerca de 30 dias depois das marcações iniciais, realizou-se nova contagem, registrando-se os novos botões emitidos.

A contagem dos frutos fixados foi feita uma semana antes do início da colheita nos pomares de 'Paluma' e 'Rica', e, quando os frutos apresentavam diâmetro $\geq 3 \mathrm{~cm}$, no pomar de 'Pedro Sato'. O tempo total decorrido entre a $1^{\mathrm{a}} \mathrm{e}$ a última contagem foi de aproximadamente 120 dias para 'Paluma' e 'Rica', e 85 dias para 'Pedro Sato'.

A partir do número total de botões florais emitidos (NB), somadas primeira e segunda contagens, e de frutos fixados (NF), calculou-se o índice de pegamento de frutos, $\mathrm{IP}=(\mathrm{NF} / \mathrm{NB}) 100$, adaptado de Rocha et al. (1990).

A comparação entre cultivares, em cada um dos anos agrícolas avaliados, foi feita através dos testes $\mathrm{F}$ e de Tukey, conforme delineamento inteiramente casualizado, com número de repetições diferentes na safra 1999-2000. Analogamente, comparou-se o pegamento de frutos em ramos novos originados da $1^{\text {a }}$ ou $2^{\text {a }}$ gema de ramos podados a partir da extremidade do ramo podado. Essa última comparação foi feita apenas na safra 2000-2001.

A análise estatística revelou a existência de diferenças significativas quanto ao índice de pegamento de frutos entre as cultivares na primeira avaliação (Tabela 1). Pela comparação de médias, apresentadas na Tabela 2, nota-se que a 'Pedro Sato' foi mais efetiva na retenção de flores emitidas $(32,3 \%)$ do que a 'Paluma' (19,9\%) e esta, mais efetiva do que a 'Rica' $(10,7 \%)$.

Na segunda avaliação, embora tenha ocorrido tendência de manutenção dos valores de índice de pegamento observados para as cultivares Paluma (17,5\%) e Rica (13,7\%), a diferença não foi significativa (Tabelas 1 e 2).

Vale ressaltar que o manejo de poda adotado no pomar de 'Pedro Sato' (época de poda) foi diferente dos demais, de forma que o florescimento e desenvolvimento dos frutos desta cultivar ocorreram mais tardiamente, sob condições climáticas diferenciadas. Isso evidencia a importância do manejo dos pomares, conforme ressaltaram Erickson (1968), Rocha et al. (1990), Pereira (1995), Agustí (1999), Araújo et al. (1999), Jutamanee et al. (2000).

Os índices aqui obtidos para as cultivares Paluma e Rica estão próximos daqueles relatados por Feldberg et al. (1997) para as mesmas cultivares, havendo, contudo, inversão na ordem dos valores apresentados por esses autores, cujo índice de pegamento da cv. Paluma $(14,4$ $\%$ ) foi menor que o da cv. Rica (19,3\%). Essa divergência pode estar relacionada a diferenças no espaçamento de plantio, manejo do pomar ou mesmo particularidades climáticas locais, não detalhadas no resumo publicado pelos referidos autores.

As três cultivares avaliadas neste estudo apresentaram índice de pegamento de frutos superior aos 6\% registrados por Singh \& Sehgal (1968) em pomares da Índia. Embora a densidade de flores possa ser importante, particularmente em espécies e cultivares que apresentem clara alternância de produção, a fixação de frutos está mais estreitamente relacionada à produção do que a densidade de flores ou o tamanho de frutos (Forshey \& Elfving, 1977; Dennis Jr., 1981).

Ao comparar-se a goiabeira com outras frutíferas perenes, como os citros, verifica-se, nas primeiras, uma maior eficiência na retenção dos botões florais até frutos maduros, pois, nos citros, os índices de pegamento de frutos registrados na literatura têm oscilado entre 0,1 e $6 \%$ (Goldschmidt \& Monselise, 1977; Agustí et al., 1982; Gravina et al., 1996). Segundo Becerra \& Guardiola (1984) e Monselise (1986), este é o fator determinante das baixas produtividades em citros. Isso reflete, ao menos em parte, a diferença produtiva das duas frutíferas; a goiabeira, de modo geral, potencialmente mais produtiva, atingindo valores de até $100 \mathrm{t} \mathrm{ha}^{-1} \mathrm{em}$ pomares comerciais, enquanto, nos pomares comerciais mais produtivos de laranjas, a colheita atinge no máximo cerca de $50 \mathrm{t} \mathrm{ha}^{-1}$.

Em mangueiras, a diferença é ainda maior, pois a retenção de flores até o estágio de frutos maduros não ultrapassa $0,01 \%$ do total emitido (Simão, 1980).

Quanto ao pegamento de frutos em ramos novos oriundos da primeira ou da segunda gema, a partir da extremidade do ramo podado, não foram observadas diferenças significativas (Tabela 3). Supõe-se que a quebra da dominância apical, em virtude da execução da poda de limpeza e produção, elimine maiores diferenças na capacidade de dreno dos ramos originados das duas primeiras gemas.

De acordo com Araujo (2000), resultados como os aqui apresentados contribuem para a caracterização de genótipos individuais que fazem parte do germoplasma da goiabeira no Brasil, enriquecendo o conjunto de informações disponíveis e permitindo a escolha dos genótipos mais adequados às diferentes necessidades de cultivo, ou em programas de melhoramento genético. 
TABELA 1 - Resumo da análise de variância do índice de pegamento de frutos (IP) em goiabeiras adultas 'Pedro Sato', 'Paluma' e 'Rica', na 1 avaliação (1999-2000) e, 'Paluma' e 'Rica', na 2a avaliação (2000-2001), cultivadas em Taquaritinga, São Paulo.

\begin{tabular}{|c|c|c|c|c|}
\hline \multirow[t]{2}{*}{ Fontes de variação } & \multicolumn{2}{|c|}{$1^{\mathrm{a} a v a l i a c ̧ a ̃ o ~}$} & \multicolumn{2}{|c|}{$2^{\mathrm{a}}$ avaliação } \\
\hline & GL & Quadrado médio & GL & Quadrado médio \\
\hline Cultivar & 2 & $515,4 * *$ & 1 & $56,2^{\mathrm{ns}}$ \\
\hline Resíduo & 59 & 46,1 & 14 & 21,9 \\
\hline C.V. $(\%)$ & \multicolumn{2}{|c|}{35,1} & \multicolumn{2}{|c|}{30,0} \\
\hline
\end{tabular}

**, ns Significativo a $1 \%$ de probabilidade e não significativo a $5 \%$ de probabilidade, respectivamente, pelo teste $\mathrm{F}$.

TABELA 2 - Índice de pegamento de frutos (IP) em goiabeiras adultas 'Pedro Sato', 'Paluma' e 'Rica', cultivadas e Taquaritinga, São Paulo. (Médias por planta).

\begin{tabular}{|c|c|c|c|c|c|}
\hline \multirow{2}{*}{$\begin{array}{c}\text { Planta } \\
\text { (repetição) }\end{array}$} & \multirow{2}{*}{$\begin{array}{l}\text { 'Pedro Sato' } \\
1 \text { a avaliação }\end{array}$} & \multicolumn{2}{|c|}{ 'Paluma' } & \multicolumn{2}{|c|}{ 'Rica' } \\
\hline & & $\mathbf{1}^{\mathrm{a}}$ avaliação & $\mathbf{2}^{\mathrm{a}}$ avaliação & $1^{\mathrm{a}}$ avaliação & $\mathbf{2}^{\mathrm{a}}$ avaliação \\
\hline 1 & 20,6 & 31,2 & 15,4 & 14,0 & 15,9 \\
\hline 2 & 32,4 & 9,2 & 23,7 & 11,1 & 9,2 \\
\hline 3 & 25,0 & 17,1 & 19,1 & 8,5 & 15,7 \\
\hline 4 & 41,9 & 9,1 & 20,2 & 8,3 & 15,6 \\
\hline 5 & 28,3 & 18,4 & 24,0 & 8,3 & 14,3 \\
\hline 6 & 32,0 & 14,8 & 8,8 & 8,9 & 10,8 \\
\hline 7 & 47,6 & 27,9 & 8,3 & 14,9 & 15,0 \\
\hline 8 & 21,8 & 12,9 & 20,3 & 11,3 & 13,3 \\
\hline 9 & 38,5 & 26,6 & & & \\
\hline 10 & 31,1 & 13,8 & & & \\
\hline 11 & 49,3 & 8,2 & & & \\
\hline 12 & 23,7 & 10,7 & & & \\
\hline 13 & 29,8 & 15,8 & & & \\
\hline 14 & 21,1 & 13,5 & & & \\
\hline 15 & 23,0 & 27,4 & & & \\
\hline 16 & 39,7 & 29,3 & & & \\
\hline 17 & 32,5 & 19,5 & & & \\
\hline 18 & 20,2 & 15,8 & & & \\
\hline 19 & 22,9 & 32,8 & & & \\
\hline 20 & 30,0 & 21,4 & & & \\
\hline 21 & 28,6 & 27,4 & & & \\
\hline 22 & 50,0 & 22,8 & & & \\
\hline 23 & 28,7 & 17,0 & & & \\
\hline 24 & 32,1 & 26,9 & & & \\
\hline 25 & 14,0 & 27,6 & & & \\
\hline 26 & 31,7 & & & & \\
\hline 27 & 45,8 & & & & \\
\hline 28 & 53,8 & & & & \\
\hline 29 & 26,8 & & & & \\
\hline$N^{\circ}$ de plantas observadas & 29 & 25 & 8 & 8 & 8 \\
\hline C.V. (\%) & 30,9 & 38,2 & 35,1 & 24,7 & 18,2 \\
\hline IP, média avaliação (\%) & 32,3 A & 19,9 В & 17,5 & $10,7 \mathrm{C}$ & 13,7 \\
\hline IP, média geral (\%) & 32,3 & & & & \\
\hline
\end{tabular}

Médias seguidas da mesma letra não diferem entre si pelo teste de Tukey, a $5 \%$ de probabilidade, na primeira avaliação.

TABELA 3 - Índice de pegamento de frutos (IP) em goiabeiras adultas 'Paluma' e 'Rica', nos ramos novos emitidos na primeira ou segunda gema do ramo podado, a partir do seu ápice, em pomares de Taquaritinga, São Paulo.

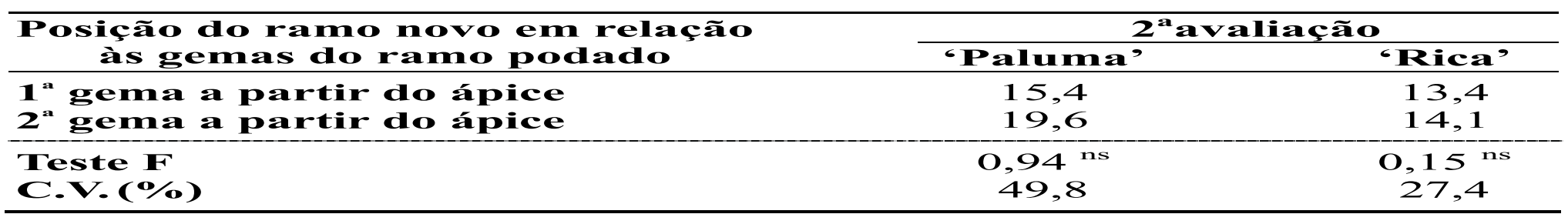

${ }^{\text {ns }}$ Não significativo a $5 \%$ de probabilidade, pelo teste F. 
Sob as condições em que o estudo foi conduzido, o índice de pegamento de frutos observado em plantas de goiabeira 'Pedro Sato', podadas no início do verão, foi de 32,3\%; já as plantas das cultivares Paluma e Rica, podadas em meados do inverno, apresentaram índice de pegamento de frutos em torno de $18,7 \%$ e $12,2 \%$, respectivamente.

\section{REFERÊNCIASBIBLIOGRÁFICAS}

AGRIANUAL: anuário da agricultura brasileira 2000. São Paulo: FNP Consultoria e Comércio, 2001. p.364-368.

AGUSTÍ, M. Floración y fructificación de los cítricos. In: SIMPÓSIO INTERNACIONAL DE FRUTICULTURA, 1., 1999, Botucatu. Anais... Botucatu: UNESP, 1999. p.161-185.

AGUSTÍ, M.; GARCÍA-MARÍ, F.; GUARDIOLA, J.L. The influence of flowering intensity on the shedding of reprodutives structures in sweet orange. Scientia Horticulturae, Amsterdam, v.17, n.4, p.343$352,1982$.

ALCÁZAR, J.R. Melhoramento genético de frutas de caroço para regiões subtropicais e de inverno ameno. In: SIMPÓSIO INTERNACIONAL DE FRUTAS DE CAROÇO: PÊSSEGO, NECTARINA E AMEIXAS, 1., 2000, Porto Alegre. Anais... Porto Alegre: UFRGS, 2000. p.17-23.

ARAUJO, D.G. Caracterização de germoplasma de cupuaçuzeiro (Theobroma grandiflorum Wield exSpreng Schum) utilizando descritores de fruto. 2000. 65f. Dissertação (Mestrado em Fitotecnia)Universidade Federal de Lavras, Lavras, 2000.

ARAÚJO, P.S.R.; MOURÃO FILHO, F.A.A.; SPOSITO, M.A. Pegamento de frutos de laranjeira- 'Pêra' em diferentes alturas na copa relacionado aos quadrantes geográficos. Scientia Agricola, Piracicaba, v.56, n.1,p.157-162, 1999.

BECERRA, S.; GUARDIOLA, J.L. Inter-relationship between flowering and fruiting in sweet orange, cultivar navelina. Proceedings of the International Society of Citriculture, v.1, p.190-194, 1984.

BUSTAN, A.; GOLDSCHMIDT, E.E.; ERNER, Y. Progress in the development of 'CITROS' - a dynamic model of citrus productivity. Acta Hortulturae, Wageningen, n.499, p.69-80, 1999.

CAMERON, J.W.; FROST, H.B. Genetics, breeding, and nucellar embryony. In: REUTHER, W.; BATCHELOR, L.D.; WEBBER, H.J. (Ed.) The citrus industry. Berkeley: University of California, 1968. v.2, p.325-370.

DAVIES, F.S.; ALBRIGO, L.G. Citrus: crop production science in horticulture 2. Wiltshire: Redwood Books, 1994. chap. 3, p.52-82.

DENNIS Jr., F.G. Apple. In: MONSELISE, S.P. (Ed). Handbook of fruit set and development. Boca Raton: CRC Press, 1986. p.1-44.

DENNIS Jr., F.G. Limiting factors in fruit set of 'Delicious' apple, Acta Horticulturae, Wageningen, n.120, p.119-124, 1981.

DONI, M.E. Florescimento e frutificação da mangueira (Mangifera indica L.) variedade Haden, 1974. 50 f. Dissertação (Mestrado em Fitotecnia)-Escola Superior de Agricultura Luiz de Queiroz, Universidade de São Paulo, Piracicaba, 1974.

ERICKSON, L.C. The general physiology of citrus, In: REUTHER, W.; BATCHELOR, L.D.; WEBBER, H.J. (Ed.) The citrus industry. Berkeley: University of California, 1968. v.2, p.86-126.
FELDBERG, N.P.; PEREIRA, F.M.; NACHTIGAL, J.C. Estudo da frutificação de goiabeira (Psidium guajava L.), cultivares Paluma e Rica. In: SIMPÓSIO BRASILEIRO SOBRE A CULTURA DA GOIABEIRA, 1., 1997, Jaboticabal. Anais... Jaboticabal, FUNEP GOIABRAS, 1997, p.174.

FORSHEY, C.G.; ELFVING, D.C. Fruit numbers, fruit size and yield relationships in 'McIntosh' apples. Journal of the American Society for Horticultural Science, Mount Vernon, v.102, n.4, p.399-402,1977.

GOLDSCHMIDT, E.E.; MONSELISE, S.P. Physiological assumptions toward the development of a Citrus fruiting model. Proceedings of the International Society of Citriculture. v.1, p.668-672, 1977.

GRAVINA, A.; ARBIZA, H.; JUAN,M.; ALMELA,V.; AGUSTÍ, M. Flowering fruiting interrelationships in 'Ellendale' tangor under the growing conditions of Spain and Uruguay. Proceedings of the International Society of Citriculture. v.2, p.1081-1085, 1996.

JUTAMANEE, K.; KRISANAPOOK, K.; PHAVAPHUTANON, L.; PICHAKUM, A. Anther dehiscence, pollen viability and pollen germination of three mango cultivars with different fruit set characters. Acta Hortulturae, Pattaya City, n.509, p.553-558, 2000.

KAVATI, R. Cultivares. In: SIMPÓSIO BRASILEIRO SOBRE A CULTURA DA GOIABEIRA, 1., 1997, Jaboticabal. Anais... Jaboticabal, FUNEP-GOIABRAS, 1997.p.1-16.

LOPES, C.R. Avanços na caracterização genética de germoplasma Arachis spp. e A. hypogeae. In: SIMPÓSIO LATINO-AMERICANO DE RECURSOS GENÉTICOS VEGETAIS, 1., 1997, Campinas. Programa e Resumos... Campinas: IAC/EMBRAPA, 1997. p.17-18.

MARCELIS, L.F.M.; HEUVELINK, E. Modelling fruit set, fruit growth and dry matter partitioning. Acta Horticulturae, Wageningen, n.499, p.39-49, 1999.

MONSELISE, S.P. Citrus. In: MONSELISE, S.P. (Ed.) Handbook of fruit set and development. Boca Raton: CRC Press, 1986. p.87-108.

NATALE, W. Diagnose da nutrição nitrogenada e potássica em duas cultivares de goiabeira (Psidium guajava $\mathrm{L}$ ), durante três anos, 1993. 149f. Tese (Doutorado em Agronomia - área de concentração em Solos e Nutrição de Plantas) - Escola Superior de Agricultura Luiz de Queiroz, Universidade de São Paulo, Piracicaba, 1993.

NATALE, W.; COUTINHO, E.L.M.; BOARETTO, A.E.; PEREIRA, F.M. Goiabeira: calagem e adubação. Jaboticabal: FUNEP, 1996. 22p.

PEREIRA, F.M. Cultura da goiabeira. Jaboticabal: FUNEP, 1995. 47p.

ROCHA, A.C.; TAVARES, E.D.; SANDRINI, M..; PAIVA, R.; CARVALHO, S.A. Época e intensidade de florescimento e pegamento de frutos segundo a distribuição pelos quadrantes em laranjeiras. Pesquisa Agropecuária Brasileira, Brasília, v.25, n.1, p.85-88, 1990.

SIMÃO, S. Botânica e biologia da mangueira. In: SIMPÓSIO BRASILEIRO SOBRE A CULTURA DA MANGUEIRA, 1., 1980, Jaboticabal. Anais... Jaboticabal: UNESP/FCAVJ, 1980. p.13-21.

SINGH, R; SEHGAL, O.P. Studies on the blossom biology of Psidium guajava L. (guava) 2, Pollen studies stigmatal receptivity pollination and fruit set. Indian Journal of Horticulture, Bangalore, v.25, p.5259,1968 . 